\title{
Anatomia comparativa da dura-máter de Sapajus libidinosus ${ }^{1}$
}

\author{
Kleber F. Pereira ${ }^{2 *}$, Vanessa A. Menezes ${ }^{2}$ e Eugênio G. de Araújo ${ }^{3}$
}

\begin{abstract}
Pereira K.F., Menezes V.A. \& Araújo E.G. 2013. [Comparative anatomy of dura-mater of Sapajus libidinosus.] Anatomia comparativa da dura-máter de Sapajus libidinosus. Pesquisa Veterinária Brasileira 33(10):1263-1268. Laboratório de Anatomia Humana e Comparativa, Curso de Ciências Biológicas, Universidade Federal de Goiás, Campus Jataí, Rodovia BR-364 Km 192, Setor Parque Industrial, Jataí, GO 75800-000, Brazil. E-mail: kpereiraufg@gmail.com

Sapajus libidinosus is a new World primate commonly known as capuchin monkey, geographically distributed throughout South America, has characteristics of restlessness and ability to manipulate tools to obtain food. This study aimed to describe the morphology of the dural sinuses of $S$. libidinosus through a comparative perspective. We used 3 adult male from the Federal University of Goiás, Brazil, fixed in aqueous $10 \%$ formaldehyde. The longitudinal fissure in the brain extends deeply and divides the organ into two left right hemispheres, The frontal and parietal lobe presents relatively smooth surface, but with well-defined gyri and sulci. In the occipital lobe, however, smooth surface aspect prevails. We observed eight sinuses of the dura mater, sagittal dorsal, ventral sagittal, transverse, straight, sigmoid, basilar and temporal which corresponds generally to the descriptions of other primates as Pan, Papio and man. Furthermore, parietal sinus similar to Saimiri sciureus and absence of sinus occipital, as in Macaca mullata, were also recorded. In the capuchin monkey, intracavernous, sphenoparietal, superior petrosal and inferior petrosal sinuses, which are described in Pan, Papio and Man, were absent. Taken together, these data point to a possible grater evolutionary proximity to man of Papio and Pan as compared to Cebus.
\end{abstract}

INDEX TERMS: Sapajus libidinosus, dura-mater, sinus venosus.

RESUMO.- O Sapajus libidinosus é um primata do novo mundo popularmente conhecido como macaco-prego, geograficamente distribuído por todo território sul-americano, possui características de inquietude e habilidade de manipulação de ferramentas para a obtenção de alimentos. Este estudo teve por objetivo descrever a morfologia dos seios da dura-máter de Sapajus libidinosus numa perspectiva comparativa. Foram utilizados um total de 3 animais adultos e machos oriundos da Universidade Federal de Goiás e fixados em solução aquosa de formaldeído $10 \%$. No encéfalo de S. libidinosus, foi possível observar a fissura lon-

\footnotetext{
${ }^{1}$ Recebido em 7 de dezembro de 2012.

Aceito para publicação em 16 de agosto de 2013.

${ }^{2}$ Docente do Curso de Ciências Biológicas, Universidade Federal de Goiás (UFG), Campus Jataí, Unidade Jatobá, BR-364 Km 192, Setor Parque Industrial, Jataí, GO 75804-020, Brasil. *Autor para correspondência: kpereiraufg@gmail.com

${ }^{3}$ Escola de Veterinária e Zootecnia, UFG, Campus Samambaia, Cx. Postal 131, Goiânia, GO 74001-970, Brasil.
}

gitudinal, profunda e que divide o mesmo em dois hemisférios, esquerdo e direito. O lobo parietal e frontal apresenta-se relativamente lisoencefálico, mas com giros e sulcos bem definidos e no lobo occipital nota-se a predominância do aspecto lisencefálico. Nota-se a presença de 8 seios da dura-máter, que corresponde de modo geral às descrições para outros primatas como Pan, Papio e homem. Notamos a presença dos seios sagital dorsal, seio sagital ventral, seio transverso, seio reto, seio sigmóide, seio temporal e seio basilar, que são semelhantes a Pan, Papio e homem, a presença do seio parietal semelhante à Saimiri sciureus e a ausência do seio occipital, assim como em Macaca mullata. No macaco prego não foram observados os seios intracavernosos, seio esfenoparietal, seio petroso superior, seio petroso inferior, sendo estes descritos em Pan, Papio e Homem. Sendo assim, estes dados demonstram uma possível maior proximidade evolutiva entre Papio e Pan em relação ao homem do que o macaco Sapajus.

TERMOS DE INDEXAÇÃO: Sapajus libidinosus, dura-máter, seio venoso. 


\section{INTRODUÇÃO}

A anatomia comparada e evolutiva está sendo cada vez mais uma fonte importante de conhecimento, pois sede a obtenção de dados não apenas para o indivíduo estudado, como também de suporte para espécies semelhantes. Além do que, o melhor entendimento da anatomia dos primatas será de grande auxilio na sua preservação e proteção, revelando que o desaparecimento de uma espécie resulta em uma carência infinita para a ciência (Marques 2008).

Sapajus libidinosus popularmente conhecido como macaco-prego (devido ao formato de sua glande peniana), pertence ao filo Chordata, classe Mammalia, ordem Primates, família Cebidae e gênero Sapajus sp. (Oppenheimir 1969). Possui hábitos generalistas, com dieta baseada em frutos e artrópodes (Terborgh 1983) é distribuído geograficamente em grande parte do continente sul americano (Hill 1962). Os animais do gênero Sapajus se destacam por apresentarem alta encefalização e capacidade de manipulação de ferramentas (Fragazy et al. 1990), como o uso de pedras para quebrar cocos ou mesmo o uso de varetas para 'pescar' formigas ou cupins, além de características de inquietude e curiosidade em seu comportamento (Aurichio 1995).

Não é de hoje que a ciência tenta especificar as diferenças anatômicas e funcionais entre o cérebro humano e o do macaco, em uma perspectiva de unificação da primeira espécie. Várias pesquisas têm sido feitas em uma análise comparativa e evolucionista, onde apesar de ainda se fazerem escassas, os estudos sobre o Cebus libidinosus em relação a outros primatas vêm aumentando consideravelmente nos últimos anos, devido a fatores como o seu fácil manuseio, relativa facilidade de se reproduzirem em cativeiro, além das equânimes semelhanças com primatas não-humanos e humanos.

Sobre as descrições morfológicas do macaco-prego, foram desenvolvidas pesquisas relativas aos músculos profundos do antebraço (Aversi-Ferreira 2005), nervos do braço (Aversi-Ferreira 2005), músculos flexores superficiais do antebraço (Aversi-Ferreira 2006), artérias do ombro (Aversi-Ferreira 2007), músculos do ombro e do braço (Aversi-Ferreira 2007), artérias da base do encéfalo (Ferreira 1997), nervos do plexo braquial (Ribeiro 2002), sulcos e giros encefálicos (Pereira-de-Paula 2010), tronco encefálico (Watanabe 1982), cerebelo (Oliveira 2007), padrão arterial do cerebelo (Ferreira \& Silva 2005), formação do sistema carótido (Siqueira Neto \& Ferreira 2002) e morfologia do plexo lombar, sacral e coccígeno, (Carvalho-Barros 2002), entretanto, sobre a organização dos envoltórios cerebrais relacionados ao crescimento do encéfalo e seus componentes de resistência e tensão, no caso as meninges encefálicas e seios da dura-máter, não foram descritas.

Para melhor conhecer sua morfologia, objetivou-se descrever as características macroscópicas dos seios da dura-máter de Sapajus libidinosus numa perspectiva comparativa.

\section{MATERIAL E MÉTODOS}

Para o presente trabalho, foram utilizados três macacos-prego (Sapajus libidinosus), machos e adultos (1-3 kg), doados pela Universidade Federal de Goiás (UFG), Campus Goiânia ao Museu de
Anatomia Humana e Comparativa, UFG, Campus Jataí, provenientes de óbito natural e armazenados em frízeres de congelamento. Mediante a aprovação do Comitê de Ética da Universidade Federal de Goiás (CoEP-UFG 81/2008), autorização do IBAMA no 15275 , os animais foram fixados e conservados em substância de formaldeído a $10 \%$ em caixas opacas em temperatura ambiente.

Foi efetuado o rebatimento dos tecidos que revestem o crânio com posterior retirada da calota craniana, de forma a manter a integridade do encéfalo e dos tecidos que o revestem, utilizando serra de aço no sentido crânio-caudal, desde a altura do osso frontal ao occipital. Em seguida procedeu-se a remoção do encéfalo da cavidade craniana cuidadosamente e procedeu-se à remoção dos envoltórios cerebrais (Pereira et al. 2010).

Todo o material foi documentado por intermédio de fotografia, através de câmera fotográfica digital, câmera Sony $\alpha$ 200-10.2 mpx, para melhor análise de sua morfologia. A denominação das estruturas foi baseada na descrição de humanos (Machado 2000) e de outros primatas (Swindler \& Wood 1973). Sempre que possível, e por analogia, as estrutura meníngeas receberam os mesmos nomes daqueles descritos para humanos e de outros primatas outrora estudados.

\section{RESULTADOS E DISCUSSÕES}

$\mathrm{Na}$ análise anatômica do encéfalo do macaco Cebus libidinosus, foram evidenciadas estruturas cerebrais não obstantes das encontradas em outros mamíferos, como nas descrições de (Hidelbrand 1995): cérebro e uma longa e profunda fissura longitudinal encefálica (Fig.1A) e o tronco encefálico (Fig.1B). As estruturas encontravam-se revestidas por camadas meníngeas, não apresentando delimitação evidente, sendo contínuas e em certos pontos as meninges apresentavam-se intimamente ligadas ao encéfalo e em outras regiões, como na abóbada craniana, estavam aderidas ao osso craniano (Fig.1B). As meninges juntamente com o crânio constituem uma proteção rígida e de crucial importância na manutenção da integridade do sistema nervoso central (Machado 2000).

$\mathrm{Na}$ análise do cérebro do Sapajus libidinosus, observou-se a fissura longitudinal (Fig.1A) que o dividia em dois hemisférios, direito e esquerdo. No lobo temporal e parietal um pequeno número de sulcos e giros se apresentavam com limites bem demarcados, sendo possível observar a presença do sulco lateral. Este sulco penetrou no lobo parietal dividindo-o em duas porções, uma superior e outra inferior. Notou-se ainda a presença do sulco central que delimitava o lobo frontal e parietal, e neste observou-se a presença do sulco pós-central e sulco lunates (profundos). 0 sulco pós-central delimitou juntamente como o sulco central o giro pós-central (Fig.2A,B), dados estes que corroboram com os achados de Pereira-de-Paula et al. (2010).

O lobo frontal e o lobo parietal do macaco-prego eram relativamente lisencefálicos com um diminuto número de giros e sulcos, aproximando das descrições para babuínos, chimpanzés e humanos (Swindler \& Wood 1973), enquanto o lobo occipital apresenta-se totalmente lisencefálico, assim como descrito em Saimiri sciureus (Barcellos et al. 2011).

No lobo frontal, nota-se a presença dos sulcos longitudinais inferiores e superiores e o sulco vertical (Fig. 2A). Segundo Pereira-de-Paula et al. (2010), estes sulcos são re- 

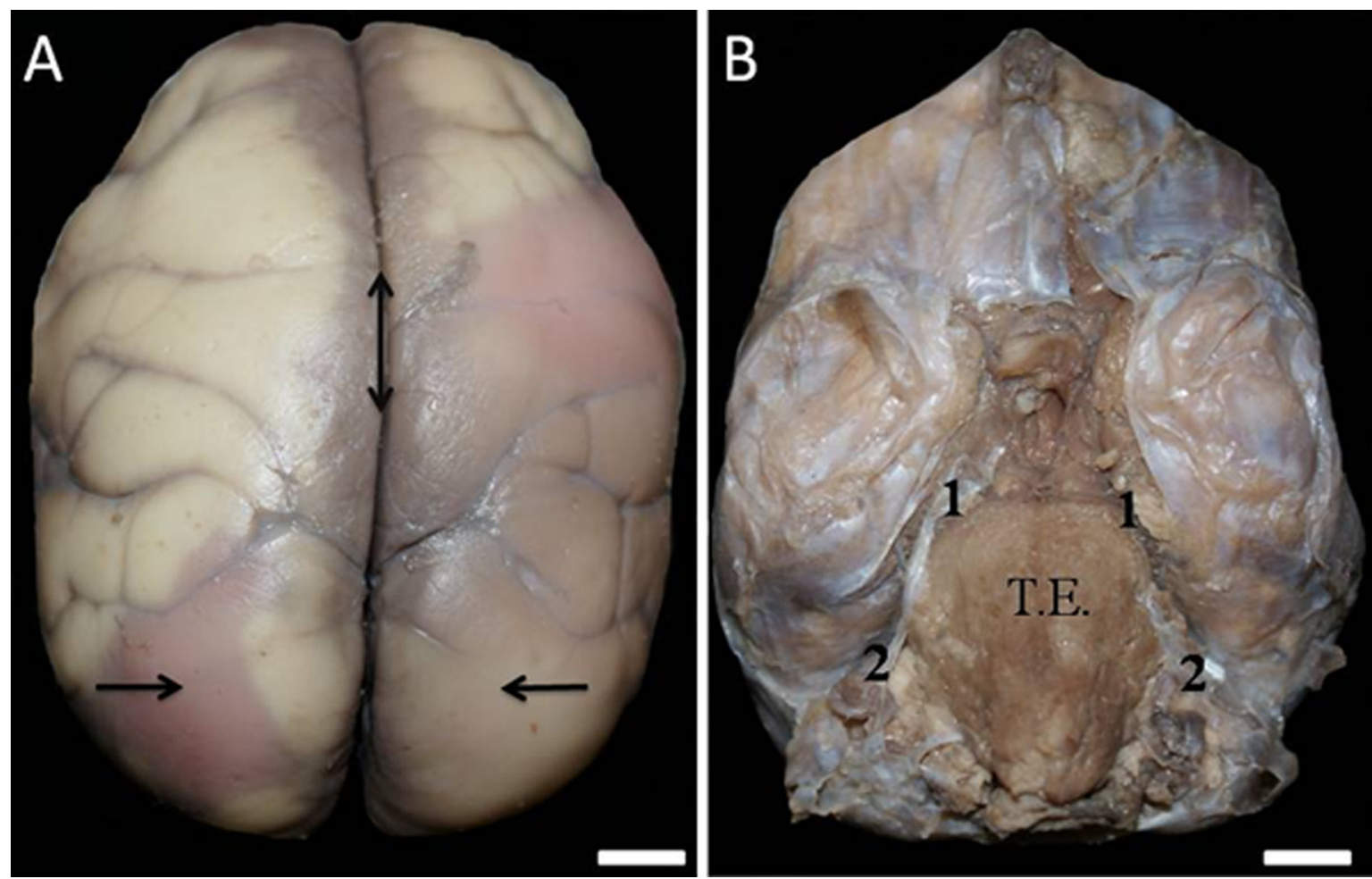

Fig.1. Fotografia do cérebro de Sapajus libidinosus evidenciando: (A) Vista dorsal: fissura longitudinal ( $\leftrightarrow$ ) e aspecto lisencefálico $(\rightarrow \leftarrow)$. (B) Vista Ventral: tronco encefálico (TE), dura-máter apresenta seios basilar (1) e seio sigmóide (2). Barra: $1 \mathrm{~cm}$.
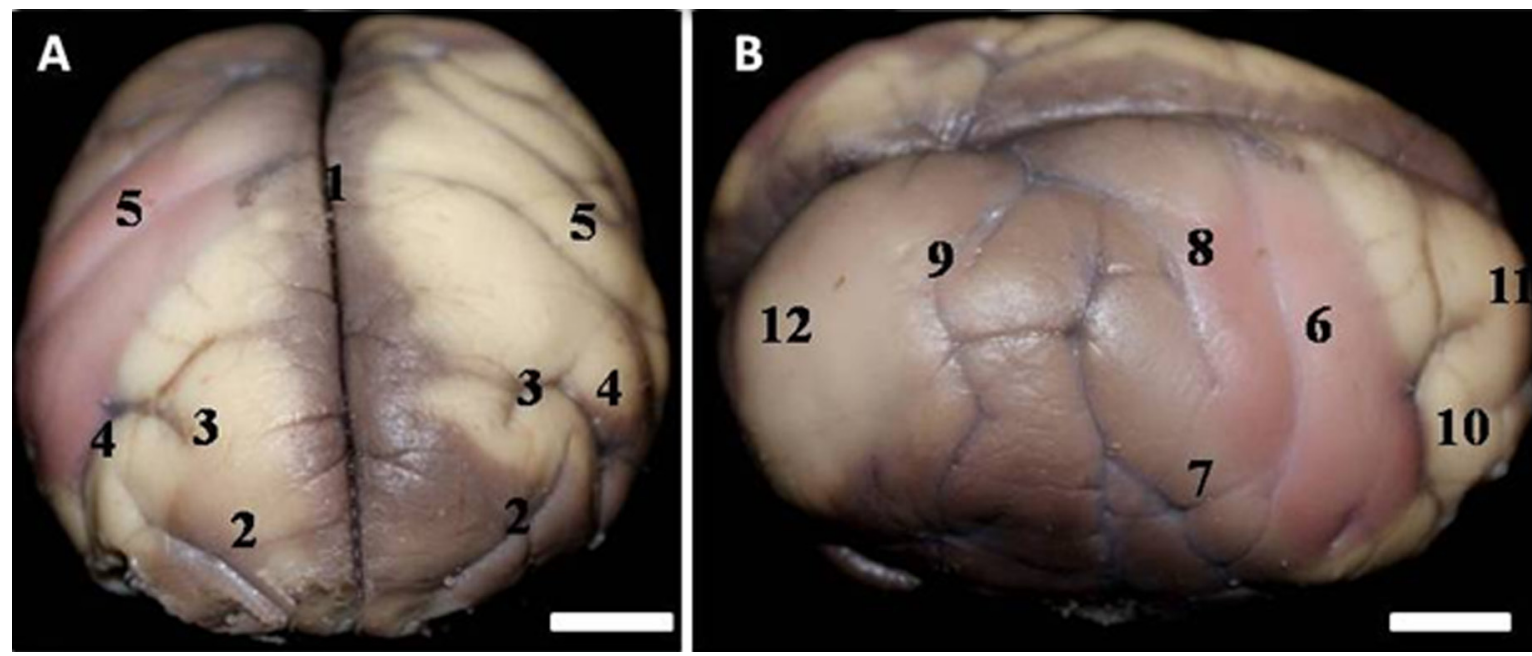

Fig.2. Cérebro de Sapajus libidinosus. (A) Fissura longitudinal mediana (1); sulco longitudinal inferior (2); sulco longitudinal superior (3); sulco vertical (4); sulco central (5). (B) Sulco central (6); sulco lateral (7); sulco pós-central (8); sulco lunates (9); sulco vertical (10); sulco longitudinal superior (11); lobo occipital lisoencefalico (12). Barra: $1 \mathrm{~cm}$.

lativamente retos e profundos, sendo o sulco longitudinal superior em posição caudal em relação ao sulco inferior, esse pequeno sulco se encontra rostral aos demais sulcos, estando localizado em uma posição próximo a de noventa graus com o sulco longitudinal superior do qual vem emergir, dados estes que corroboram com nossos achados (Fig.2A).

Observamos na dura-máter estruturas de compartimentação do encéfalo, como foice do cérebro, que entremeia profundamente a fissura longitudinal do cérebro e a tenda do cerebelo (Fig.3C). A tenda estava situada em posição caudal, entre o lobo occipital e o cerebelo, separando o cérebro e o cerebelo, dividindo a cavidade craniana em um compartimento superior, supratentorial e outro inferior, infratentorial (Machado 2000). Essas estruturas são descritas por Schwarze \& Schroder (1972), Testut \& Jacob (1951) no homem, Ferreira (2005) para Bradypus e Swindler \& Wood (1973) para Pan e Papio.

A dura-máter é descrita como uma formação membranácea ricamente inervada, sendo que, em determinadas 

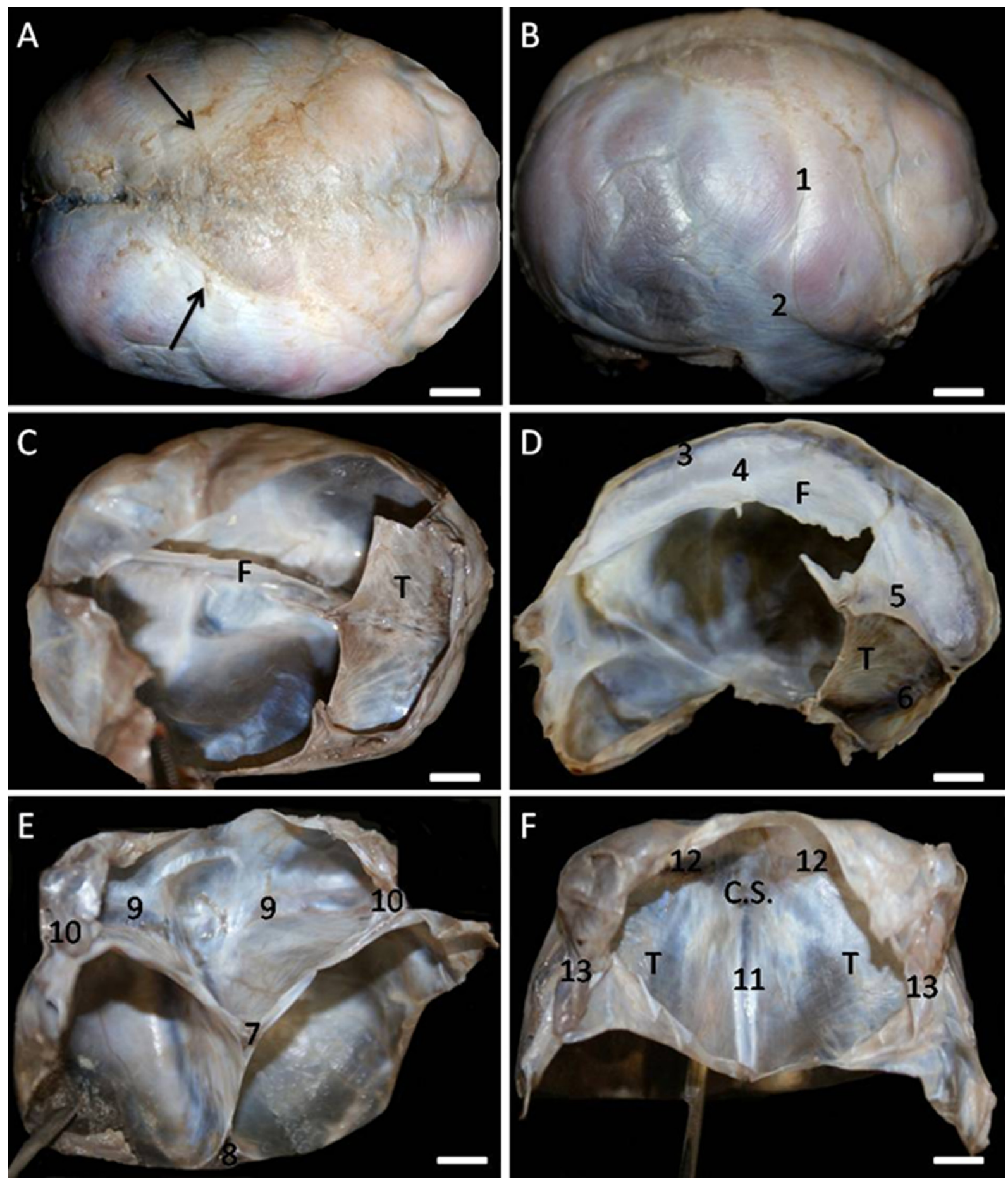

Fig.3. Os seios da dura-máter de Sapajus libidinosus. (A) Vista dorsal: encéfalo revestido pelas meninges. (B) Vista lateral: seio parietal (1) e seio temporal (2). (C) Vista ventral expõe foice do cérebro (FO), tentório do cerebelo (T). (D) Vista lateral do corte longitudinal apresenta seio sagital superior (3), seio sagital inferior (4), seio reto (5), seio transverso (6), foice do cérebro (FO) e tentório do cerebelo (T). (E) Vista posterior do corte transverso expõe seio reto (7), seio sagital superior (8), seio transverso (9), seio sigmóide (10). (F) Vista posterior ventral do corte transverso da dura-máter. Tentório do cerebelo (T), Seio reto (11), Seio Transverso (12), Confluência dos seios (CS) e Seio Sigmóide (13). Barra: $1 \mathrm{~cm}$.

áreas, os dois folhetos separam-se, delimitando cavidades (Machado 2000). A terminologia anatômica que foi utilizada para nomeação destas estruturas no Cebus libidinosus é a mesma para o homem, devido à escassez de fontes sobre o referido assunto. Os seios venosos da dura-máter são ca- nais venosos encontrados entre as camadas de dura-máter no cérebro. Em nossa pesquisa, identificamos em Cebus libidinosus um total de oito seios venosos, cuja denominação, situação e trajeto, origem e destino são explicitados no Quadro 1. 
Quadro 1. Descrição dos respectivos seios da dura-máter no Sapajus libidinosus, em relação à sua situação e trajeto, origem e destino

\begin{tabular}{|c|c|c|c|}
\hline $\begin{array}{l}\text { Identificação dos seios } \\
\text { da dura-máter }\end{array}$ & Situação e trajeto & Origem & Destino \\
\hline 1- Seio transverso & $\begin{array}{l}\text { Bilateral, percorre a inserção da } \\
\text { tenda do cerebelo }\end{array}$ & Confluência dos seios & Seio basilar \\
\hline 2- Seio sagital dorsal & $\begin{array}{l}\text { Mediano, ímpar percorre a inser- } \\
\text { ção da foice do cérebro }\end{array}$ & $\begin{array}{l}\text { Placa cribiforme, no } \\
\text { septo nasal }\end{array}$ & Confluência dos seios \\
\hline 3- Seio Sagital ventral & $\begin{array}{l}\text { Ímpar, situa-se na } \\
\text { margem livre da foice do cérebro }\end{array}$ & $\begin{array}{l}\text { No início do Seio sa- } \\
\text { gital dorsal }\end{array}$ & Seio reto \\
\hline 4- Seio reto & $\begin{array}{l}\text { Ímpar, situa-se a o longo da linha } \\
\text { de união entre a foice do cérebro } \\
\text { e a tenda do cerebelo }\end{array}$ & $\begin{array}{l}\text { Seio sagital ventral e } \\
\text { veia cerebral magna }\end{array}$ & Confluência dos seios \\
\hline 5- Seios temporais & $\begin{array}{l}\text { Bilateral, percorre a borda rostral } \\
\text { da meninge no lobo temporal }\end{array}$ & $\begin{array}{l}\text { Face lateral da dura- } \\
\text {-máter }\end{array}$ & $\begin{array}{l}\text { Conflui para o } \\
\text { seio basilar }\end{array}$ \\
\hline 6- Seios parietais & Par, percorre todo o lobo parietal & Seio sagital dorsal & Seio petroso superior \\
\hline 7- Seio basilar & $\begin{array}{l}\text { Bilateral, presente em cada lado } \\
\text { da região da hipófise }\end{array}$ & $\begin{array}{l}\text { Confluência dos seios } \\
\text { sigmóide e transverso }\end{array}$ & Plexo venoso epidural \\
\hline 8- Seio sigmóide & $\begin{array}{l}\text { Bilateral, em forma de } S \text {, corre na } \\
\text { lâmina basal da meninge }\end{array}$ & Seio Transverso & $\begin{array}{l}\text { Forame jugular, na } \\
\text { veia jugular interna }\end{array}$ \\
\hline
\end{tabular}

Os seios observados foram: 1) seio sagital dorsal (Fig.3D) - ímpar, dorsal, mediano, paralelo à foice do cérebro, termina na confluência dos seios. (Fig.3F); 2) Seio sagital ventral (Fig.3D) - ímpar, mediano, percorre a foice do cérebro terminando no seio reto; 3) Seio Transverso (Fig.3E) - par, dispõe-se de cada lado da inserção da tenda do cerebelo; 4) Seio reto (Fig.3F) - ímpar localiza-se entre a foice do cérebro e a tenda do cerebelo; 5) Seio sigmóide (Fig.3F) - par, possui forma de $\mathrm{S}$ sendo uma forma de continuação do seio transverso; 6) Seio temporal (Fig.3B) - bilateral, inferior ao seio parietal; 7) Seio basilar (Fig.1B) - bilateral, superior ao seio sigmóide, apresenta-se ao lado da ponte do tronco encefálico; e 8) Seio parietal (Fig.3B) bilateral, dispõe-se acima do seio temporal, se ligando ao seio sagital superior.

A morfologia da dura-máter de Sapajus libidinosus revelou particularidades como a presença do seio parietal, que foi reconhecido na literatura em Saimiri Siureus (Barcellos et al. 2011). Observamos a presença do seio transverso e seio dorsal, sendo o segundo uma continuação do primeiro. 0 seio dorsal emergiu da confluência dos seios, dados que corroboram com as descrições de Ferreira (2005), para Bradypus. No entanto, os Sapajus libidinosus estudados não apresentaram os seios orbículo-olfatório, para-sagital cerebelar e inter-transverso, como observado em Bradypus por Ferreira (2005). A presença destes seios em Bradypus, principalmente do seio orbículo-olfatório pode estar associado ao nicho ecológico deste animal, que tem como principal sentido integrador com o ambiente, o olfato (Jollie 1980), o que resultaria em uma maior necessidade de suprimento sanguíneo.

De acordo com Swindler \& Wood (1973), o seio sagital dorsal, ventral e seio reto estão presentes em Papio, Pan e Homem, assim como em Sapajus. Entretanto, o seio occipital, não foi observado em nossos achados, assim como na Macaca mullata (Kassel \& Langefitt 1965), sendo observado apenas em Papio (Swindler \& Wood 1973).

Em Pan e no homem, o seio petroso superior e seio cavernoso são descritos como conectados ao seio transverso, contudo no Sapajus libidinosus foi evidenciado apenas a pre- sença do seio transverso. Segundo Swidler \& Wood (1973), em Papio o seio sigmóide não possui a forma tortuosa em forma de S, descrito nas demais espécies, como Pan, homem e Sapajus. A morfologia da confluência dos seios é descrita como semelhante para Pan, Papio, Homem e Sapajus.

Os dados morfológicos acerca da dura-máter verificados neste estudo aproximam-se das descrições para humanos (Machado 2002), entretanto, há diferenciações que os distanciam como a presença do seio parietal e a ausência do seio cavernoso. Em Sapajus libidinosus não foram observados os seios intracavernosos, seio esfenoparietal, seio petroso superior, seio petroso inferior que são encontrados em Pan, Papio e Homem. Tais dados demonstram uma possível maior proximidade evolutiva entre Papio e Pan em relação ao homem do que o macaco Sapajus.

\section{CONCLUSÕES}

A partir dos resultados obtidos, podemos inferir que a dura-máter de Sapajus libidinosus reveste o encéfalo, possuindo estruturas de compartimentação como foice do cérebro, tenda do cerebelo e um conjunto de 8 seios da dura-máter.

Observamos que o lobo frontal e parietal são relativamente lisencefálicos com um diminuto número de giros e sulcos, aproximando das descrições para babuínos, chimpanzés e humanos e o lobo occipital apresenta-se totalmente lisencefálico, assim como descrito em Saimiri sciureus.

A organização dos seios da dura-máter de Sapajus libidinosus corresponde de modo geral às descrições para outros primatas como Pan, Papio e homem.

Notamos a presença dos seios sagital dorsal, seio sagital ventral, seio transverso, seio reto, seio sigmóide, seio temporal e seio basilar, que são semelhantes a Pan, Papio e homem, a presença do seio parietal semelhante a Saimiri sciureus e a ausência do seio occipital, assim como em $M a-$ caca mullata.

No macaco prego não foram observados os seios intracavernosos, seio esfenoparietal, seio petroso superior, seio petroso inferior, sendo estes descritos em Pan, Papio e Homem. 
Sendo assim, estes dados demonstram uma possível maior proximidade evolutiva entre Papio e Pan em relação ao homem do que o macaco Sapajus.

\section{REFERÊNCIAS}

Auricchio P. 1995. Primatas do Brasil. Terra Brasilis, São Paulo. 35p.

Aversi-Ferreira T.A., Aversi-Ferreira R.A.G.M.F., Silva Z., Gouvêa-e-Silva L.F. \& Penha-Silva N. 2005. Estudo anatômico de músculos profundos do antebraço de Cebus apella (Linnaeus, 1766). Acta Scient. Biol. Sci. 27:297301.

Aversi-Ferreira T.A., Lima-e-Silva M.S., Pereire-de-Paula J., Gouvêa-e-Silva L.F. \& Penha-Silva N. 2005. Anatomia comparativa dos nervos do braço de Cebus apela: descrição do músculo dorsoepitroclear. Acta Scient. Biol. Sci. 27:291-296

Aversi-Ferreira T.A., Vieira L.G., Pires R.M., Silva Z. \& Penha-Silva N. 2006. Estudo anatômico dos músculos flexores superficiais do antebraço no macaco Cebus apella. Biosci. J. 22:139-144.

Aversi-Ferreira T.A., Pereira-de-Paula J., Lima-e-Silva M.S. \& Silva Z. 2007. Anatomy of the arteries of the arm of Cebus libidinosus (Rylands et al. 2000) monkeys. Acta Sci. Biol. Sci. 29:247-254.

Aversi-Ferreira T.A., Pereira-de-Paula J., Prado Y.C.L., Lima-e-Silva M.S. \& Mata J.R. 2007. Anatomy of the shoulder and arm muscles of Cebus libidinosus. Braz. J. Morphol. Sci. 24:63-74.

Aversi-Ferreira T.A., Pereira-de-Paula J., Lima-e-Silva M.S., Prado Y.C.L. \& Silva Z. 2007. Estudo anatômico das artérias do ombro de Cebus libidinosus (Rylands, 2000; Primates - Cebidae). Ciênc. Anim. Bras. 8:272284.

Barcellos J.F.M., Branco E., Fioretto E., Imbeloni A.A., Muniz J.A.P.C. \& Lima A.R. 2011. Macroscopic aspects of Saimiri sciureus dura-mater. Pesq. Vet. Bras. 31:7-10.

Carvalho-Barros R.A. 2002. Estudo anatômico do plexo lombar, sacral e coccígeno do macaco Cebus apela: origem, composição e nervos resultantes. Tese de Mestrado em Anatomia dos Animais Domésticos, Faculdade de Medicina Veterinária e Zootecnia, Universidade de São Paulo, São Paulo, SP. 146p.

Ferreira J.R., Oliveira V.L., Oliveira K.M. \& Oliveira M.A. 2005. Contribuição ao estudo anatômico da dura-máter do Bradypus torquatus (Linnaeus, 1758) e Bradypus variegatus (Schinz, 1825). Braz. J. Vet. Res. Anim. Sci. 42:381-387.

Ferreira J.R. 1997. 0 estudo anatômico das artérias da base do encéfalo do macaco-prego (Cebus apela Lineaus, 1766). Tese de Doutorado em Ciências, Faculdade de Medicina Veterinária e Zootecnia, Universidade de São Paulo, São Paulo, SP. 159p.

Ferreira J.R. \& Silva R.A. 2005. 0 padrão arterial do cerebelo do macaco-prego (Cebus apela Linnaeus, 1766). Veterinária Notícias, Uberlândia, 11:11-18.
Fragaszy D.M., Visalberghi E. \& Robinson J.G. 1990. Variability and adaptability in the genus Cebus. Folia Primatologica 54:1148-1158.

Hildebrand M. 1995. Análise da Estrutura dos Vertebrados. Atheneu, São Paulo. 642p.

Hill W.C.O. 1960. Primates comparative anatomy and taxonomy. IX Cebidae, Part A. University Press, Edinburgh, p.63-282p.

Jollie M. 1980. Chordate Morphology. Publishing Chapman and Hevl, London. 508p.

Kassell N.F. \& Langefill T.W. 1965. Variations in the circle of Willis in Macaca mulatta. Anat. Rec. 162:257-264.

Machado A.B.M. 2000. Neuroanatomia Funcional. $2^{2}$ ed. Atheneu, São Paulo. $363 \mathrm{p}$.

Marques K.L.S. 2008. Associação de emissões vocais de macacos-prego ( $C e$ bus apella, Primate, Cebidae) a contextos comportamentais em cativeiro. Dissertação de Mestrado, Universidade Federal do Pará, Belém. 56p.

Oliveira W.G. 2007. Estudo anatômico do cerebelo do macaco-prego ( $\mathrm{Ce}$ bus apella Linneaus, 1758). Revta Biotemas 20:49-58.

Oppenheimer J.R. 1968. Behavior and ecology of the White faced monkey, Cebus capucinus, on Barro Colorado Island. PhD Thesis, University of Illinois, Urbana. $179 \mathrm{p}$.

Perera K.F., Lima V.M., Conegero C.I. \& Chopard R.P. 2010. Histomorfometria das meninges encefálicas de ratos Wistar em diferentes faixas etárias. Pesq. Vet. Bras. 30:996-1002.

Pereira-de-Paula J., Prado Y.C.L., Tomaz C. \& Aversi-Ferreiva T.A. 2010. Anatomical study of the main sulci and gyri of the Cebus Libidinosus brain (Rylands, 2000). Neurobiologia 73:65-78.

Ribeiro A.R. 2002. Estudo anatômico do Plexo braquial do macaco Cebus apela: origem, composição e nervos resultantes. Tese de Mestrado em Anatomia dos Animais Domésticos, Faculdade de Medicina Veterinária e Zootecnia, Universidade de São Paulo, São Paulo, SP. 146p.

Schwarze E. \& Schroder L. 1972. Compendio de Anatomía Veterinária. Acribia, Zaragoza. 247p.

Siqueira Neto E.G.B. \& Ferreira J.R. 2002. Estudo anatômico da origem e distribuição dos ramos corticais das artérias cerebrais caudais do encéfalo do macaco prego (Cebus apella Linnaeus, 1766). Acta Scientiarum 24:639-646p.

Swindler D.R. \& Wood C.D. 1973. An Atlas of Primate Gross Anatomy. University of Washington Press, Seattle, Washington. 149p.

Terborgh J. 1983. Five new world primates: a study in comparative ecology. Princeton University Press, Princeton, New Jersey. 280p.

Testut L. \& Jacob 0. 1951. Compendio de Anatomia Topografica. Editora Salvat, Rio de Janeiro. 481p.

Watanabe I. 1982. Comparative study of the medula oblongata, pons, mesencephalon and cerebellum of the tufted capuchim Cebus apella Linnaeus, 1758. Revta Odont. Unesp 11:13-25. 